\title{
ARITHMETIC OF DEL PEZZO SURFACES
}

\author{
ANTHONY VÁRILLY-ALVARADO
}

\section{INTRODUCTION}

These notes were written to accompany a mini-course delivered during the conference "Arithmetic of Surfaces," which was held at the Lorentz Center in Leiden, during October, 2010. The mini-course was aimed at graduate students and consisted of three lectures. These notes are a faithful transcript of the material we went over, with some added details and references. I have resisted the temptation to add any more material. In particular, many aspects of the arithmetic of del Pezzo surfaces are not treated here (most notably perhaps the uniqueness of Brauer-Manin obstructions).

In preparing this document, I used the "background material" chapters of my doctoral dissertation [VA09] quite freely (especially for the first and third lectures); these chapters were not meant for publication.

Acknowledgements. I thank the conference organizers (Hendrik Lenstra, Cecilia Salgado, Lenny Taelman and Ronald van Luijk) for inviting me to give this mini-course and for their hospitality in Leiden. I also thank the staff at the Lorentz Center for all their help and professionalism. Finally, I thank Jean-Louis Colliot-Thélène for comments on these notes.

\section{Lecture 1: Geometry of Del Pezzo surfaces}

1.1. Guiding questions in diophantine geometry. Let $k$ be a global field, i.e., a finite extension of $\mathbb{Q}$ or $\mathbb{F}_{p}(t)$ for some prime $p$, let $\mathbf{A}_{k}$ denote its ring of adèles, and let $X$ be a smooth projective geometrically integral variety over $k$. Generally speaking, diophantine geometers seek to "describe" the set $X(k)$ of $k$-rational points of $X$. For example, we are interested in determining whether $X(k)$ is empty or not. If $X(k) \neq \emptyset$, then we may further want to know something about the qualitative nature of $X(k)$ : is it dense for the Zariski topology of $X$ ? Is the image of the natural embedding $X(k) \hookrightarrow X\left(\mathbf{A}_{k}\right)$ dense for the adèlic topology? If not, can we account for the paucity of $k$-rational points? We may also pursue a more quantitative study of $X(k)$. For instance, we might try to prove asymptotic formulas for the number of $k$-points of bounded height on some special Zariski-open subset of $X$.

On the other hand, if $X(k)=\emptyset$, then we might try to account for the absence of $k$-rational points. For example, the existence of embeddings $X(k) \hookrightarrow X\left(k_{v}\right)$ for every completion $k_{v}$ of $k$ shows that a necessary condition for $X$ to have a $k$-rational point is

$$
X\left(k_{v}\right) \neq \emptyset \text { for all completions } k_{v} \text { of } k \text {. }
$$


We say that $X$ is locally soluble whenever (1) is satisfied. Whenever checking (1) suffices to show that $X(k) \neq \emptyset$, we say that $X$ satisfies the Hasse principle ${ }^{1}$. Many classes of varieties, such as quadrics, satisfy the Hasse principle.

Perhaps the first known counterexample to the Hasse principle is due to Lind and Reichardt, who show that the genus 1 plane curve over $\mathbb{Q}$ with affine model given by $2 y^{2}=$ $x^{4}-17$ is locally soluble, but lacks $\mathbb{Q}$-rational points; see [Lin40, Rei42]. Failures of the Hasse principle are often explained by the presence of cohomologically flavored obstructions, such as the Brauer-Manin obstruction. These kinds of obstructions may also produce examples of varieties $X$ as above, with $X(k) \neq \emptyset$, for which the embedding $X(k) \hookrightarrow X\left(\mathbf{A}_{k}\right)$ is not dense.

Notation. The following notation will remain in force throughout. First, $k$ denotes a field, $\bar{k}$ is a fixed algebraic closure of $k$, and $k^{s} \subseteq \bar{k}$ is the separable closure of $k$ in $\bar{k}$. If $k$ is a global field then we write $\mathbf{A}_{k}$ for the adèle ring of $k, \Omega_{k}$ for the set of places of $k$, and $k_{v}$ for the completion of $k$ at $v \in \Omega_{k}$. By a $k$-variety $X$ we mean a separated scheme of finite type over $k$ (we will omit the reference to $k$ when it can cause no confusion). If $X$ and $Y$ are $S$-schemes then we write $X_{Y}:=X \times_{S} Y$. However, if $Y=\operatorname{Spec} A$ then we write $X_{A}$ instead of $X_{\operatorname{Spec} A}$. A $k$-variety $X$ is said to be nice if it is smooth, projective and geometrically integral. If $T$ is a $k$-scheme, then we write $X(T)$ for the set of $T$-valued points of $X$. If, however, $T=\operatorname{Spec} A$ is affine, then we write $X(A)$ instead of $X(\operatorname{Spec} A)$.

1.2. Birational invariance and a theorem of Iskovskikh. Let $X$ be a nice $k$-variety. Many properties of $X(k)$, such as "being nonempty," depend only on $X$ up to birational equivalence, as follows.

Existence of a smooth $k$-point. The Lang-Nishimura lemma guarantees that if $X^{\prime}-\rightarrow X$ is a birational map between proper integral $k$-varieties then $X^{\prime}$ has a smooth $k$-point if and only if $X$ has a smooth $k$-point; see [Lan54, Nis55]. We give a short proof here due to Kollár and Szabó [RY00, Proposition A.6].

Lemma 1.1 (Lang-Nishimura). Let $k$ be a field, and let $f: X^{\prime} \rightarrow-\rightarrow$ be a rational map of $k$-schemes. Assume that $X^{\prime}$ has a smooth $k$-point and that $X$ is proper. Then $X(k) \neq \emptyset$.

Proof. We use induction on $n:=\operatorname{dim} X^{\prime}$. The case $n=0$ is clear. Let $x$ be a smooth $k$-point of $X^{\prime}$. Consider the blow-up $\mathrm{Bl}_{x} X^{\prime}$ of $X^{\prime}$ at $x$ with exceptional divisor $E \cong \mathbb{P}_{k}^{n-1}$ and the composition

$$
\mathrm{Bl}_{x} X^{\prime} \rightarrow X^{\prime} \rightarrow X
$$

By the valuative criterion of properness, this composition is defined outside a set of codimension at least 2 , so the restricting to $E$ we obtain a rational map $E \rightarrow X$. Now $X(k) \neq \emptyset$ by induction. (Note that induction is only necessary in the case where $k$ is finite.)

Zariski density of $k$-rational points. If $X, X^{\prime}$ are two nice birationally equivalent $k$ varieties, then $X(k)$ is Zariski dense in $X$ if and only if $X^{\prime}(k)$ is Zariski dense in $X^{\prime}$ : the key point to keep in mind is that any two nonempty open sets in the Zariski topology have nonempty intersection.

\footnotetext{
${ }^{1}$ Many authors refer only to the Hasse principle in the context of a class $\mathcal{S}$ of varieties and say that $\mathcal{S}$ satisfies the Hasse principle if for every $X \in \mathcal{S}$, the implication $X\left(k_{v}\right) \neq \emptyset$ for all $v \in \Omega_{k} \Longrightarrow X(k) \neq \emptyset$ holds.
} 
Weak approximation. Let $X$ be a geometrically integral variety over a global field $k$. We say that $X$ satisfies weak approximation if the diagonal embedding

$$
X(k) \hookrightarrow \prod_{v \in \Omega_{k}} X\left(k_{v}\right)
$$

is dense for the product of the $v$-adic topologies. If $X$ is a nice $k$-variety then $X\left(\mathbf{A}_{k}\right)=$ $\prod_{v} X\left(k_{v}\right)$, the latter considered with the product topology of the $v$-adic topologies; see [Sko01, pp. 98-99]. In this case $X$ satisfies weak approximation if the image of the natural map $X(k) \hookrightarrow X\left(\mathbf{A}_{k}\right)$ is dense for the adèlic topology. Note also that if $X$ does not satisfy the Hasse principle, then automatically $X$ does not satisfy weak approximation.

Lemma 1.2. If $X$ and $X^{\prime}$ are smooth, geometrically integral and birationally equivalent varieties over a global field $k$, then $X^{\prime}$ satisfies weak approximation if and only if $X$ satisfies weak approximation.

Sketch of proof. It is enough to prove the lemma in the case $X^{\prime}=X \backslash W$, where $W$ is a proper closed subvariety of $X$, i.e., $X^{\prime}$ is a dense open subset of $X$. Then, if $X$ satisfies weak approximation, then clearly so does $X^{\prime}$. On the other hand, by the $v$-adic implicit function theorem, the set $X^{\prime}\left(k_{v}\right)$ is dense in $X\left(k_{v}\right)$; see [CTCS80, Lemme 3.1.2]. Suppose that $X^{\prime}$ satisfies weak approximation and let $\left(x_{v}\right) \in \prod_{v} X\left(k_{v}\right)$ be given. Choose $\left(y_{v}\right) \in \prod_{v} X^{\prime}\left(k_{v}\right) \subseteq$ $\prod_{v} X\left(k_{v}\right)$ as close as desired to $\left(x_{v}\right)$ for the product topology. By hypothesis, there is a rational point $y \in X^{\prime}(k)$ whose image in $\prod_{v} X^{\prime}\left(k_{v}\right)$ is arbitrarily close to $\left(y_{v}\right)$; then $y$ is also close to $\left(x_{v}\right)$, and $X$ satisfies weak approximation.

Hasse principle. If $k$ is a global field, and if $X, X^{\prime}$ are two nice birationally equivalent $k$-varieties, then $X$ satisfies the Hasse principle if and only if $X^{\prime}$ satisfies the Hasse principle: this follows from two applications of the Lang-Nishimura lemma.

It is thus natural to ask the qualitative questions of $\$ 1.1$ in the context of a fixed birational class for $X$. In particular, we will fix the dimension of $X$. We will consider these questions only for nice surfaces. In addition, we require that $X$ be geometrically rational, i.e., $X \times_{k} \bar{k}$ is birational to $\mathbb{P}_{\bar{k}}^{2}$. The reason for this last restriction is the existence of the following beautiful classification theorem due to Iskovskikh, which describes the possible birational classes for $X$.

Theorem 1.3 ([Isk79, Theorem 1]). Let $k$ be a field, and let $X$ be a smooth projective geometrically rational surface over $k$. Then $X$ is $k$-birational to either a del Pezzo surface of degree $1 \leq d \leq 9$ or a rational conic bundle.

Remark 1.4. It is possible for $X$ as in Theorem 1.3 to be $k$-birational to both a del Pezzo surface and a rational conic bundle. More precisely, a rational conic bundle is birational to a minimal del Pezzo surface if and only if $d=1,2$ or 4 and there are two distinct representations of $X$ as a rational conic bundle; see [Isk79, Theorems 4 and 5].

1.3. Del Pezzo surfaces. In light of Theorem 1.3, we take a moment to review the definition and some basic properties of del Pezzo surfaces. In this section, we work over an arbitrary field $k$.

We begin by recalling some basic facts and setting some notation. If $X$ is a nice surface, then there is an intersection pairing on the Picard group $(\cdot, \cdot)_{X}: \operatorname{Pic} X \times \operatorname{Pic} X \rightarrow \mathbb{Z}$; see [Kle05, Appendix B]. We omit the subscript on the pairing if no confusion can arise. 
For such an $X$, we identify $\operatorname{Pic}(X)$ with the Weil divisor class group (see [Har77, Corollary II.6.16]); in particular, we will use additive notation for the group law on Pic $X$. If $X$ is a nice $k$-variety, then we write $K_{X}$ for the class of the canonical sheaf $\omega_{X}$ in Pic $X$; the anticanonical sheaf of $X$ is $\omega_{X}^{\otimes-1}$. An exceptional curve on a smooth projective $k$-surface $X$ is an irreducible curve $C \subseteq X_{\bar{k}}$ such that $(C, C)=\left(K_{X}, C\right)=-1$. By the adjunction formula (see [Ser88, IV.8, Proposition 5]), an exceptional curve on $X$ has arithmetic genus 0, and hence it is $\bar{k}$-isomorphic to $\mathbb{P}_{\bar{k}}^{1}$.

Definition 1.5. A del Pezzo surface $X$ is a nice $k$-surface with ample anticanonical sheaf. The degree of $X$ is the intersection number $d:=\left(K_{X}, K_{X}\right)$.

If $X$ is a del Pezzo surface then the Riemann-Roch theorem for surfaces and Castelnuovo's rationality criterion show that $X$ is geometrically rational. Moreover, $X_{k^{s}}$ is isomorphic to either $\mathbb{P}_{k^{s}}^{1} \times \mathbb{P}_{k^{s}}^{1}$ (in which case $d=8$ ), or the blow-up of $\mathbb{P}_{k^{s}}^{2}$ at $r \leq 8$ distinct closed points (in which case $d=9-r)$; this is the content of Theorem 1.6 below. In the latter case, the points must be in general position: this condition is equivalent to ampleness of the anticanonical class on the blown-up surface; see [Dem80, Théorème 1, p. 27].

1.4. Del Pezzo surfaces are separably split. Throughout this section, $k$ denotes a separably closed field and $\bar{k}$ a fixed algebraic closure of $k$. A collection of closed points in $\mathbb{P}^{2}(k)$ is said to be in general position if no 3 points lie on a line, no 6 points lie on a conic, and no 8 points lie on a singular cubic, with one of the points at the singularity. Our goal is to prove the following strengthening of [Man74, Theorem 24.4].

Theorem 1.6. Let $X$ be a del Pezzo surface of degree $d$ over $k$. Then either $X$ is isomorphic to the blow-up of $\mathbb{P}_{k}^{2}$ at 9 -d points in general position in $\mathbb{P}^{2}(k)$, or $d=8$ and $X$ is isomorphic to $\mathbb{P}_{k}^{1} \times \mathbb{P}_{k}^{1}$.

We need two results of Coombes, as follows.

Proposition 1.7 ([Coo88, Proposition 5]). Let $f: X \rightarrow Y$ be a birational morphism of smooth projective surfaces over $k$. Then $f$ factors as

$$
X=X_{0} \rightarrow X_{1} \rightarrow \cdots \rightarrow X_{r}=Y
$$

where each map $X_{i} \rightarrow X_{i+1}$ is a blow-up at a closed $k$-point of $X_{i+1}$.

The above proposition is well-known if we replace $k$ with $\bar{k}$. The main step in the proof of Proposition 1.7 is to show that the blow-up at a closed point whose residue field is a nontrivial purely inseparable extension of $k$ cannot give rise to a smooth surface. Using Iskovskikh's classification theorem (Theorem 1.3), Coombes deduces the following proposition.

Proposition 1.8 ([Coo88, Proposition 7]). The minimal smooth projective rational surfaces over $k$ are $\mathbb{P}_{k}^{2}$ and the Hirzebruch surfaces $\mathbb{F}_{n}:=\mathbb{P}\left(\mathscr{O}_{\mathbb{P}_{k}^{1}} \oplus \mathscr{O}_{\mathbb{P}_{k}^{1}}(n)\right)$, where either $n=0$ or $n \geq 2$.

Finally, we need the following lemma.

Lemma 1.9 ([Man74, Theorem 24.3(ii)]). Let X be a del Pezzo surface over an algebraically closed field. Then every irreducible curve with negative self-intersection is exceptional. 
Proof. Let $C \subset X$ be an irreducible curve with $(C, C)<0$, and let $p_{a}(C)$ denote its arithmetic genus. Since $-K_{X}$ is ample, we have $\left(C,-K_{X}\right)>0$. On the other hand, by the adjunction formula we know that

$$
2 p_{a}(C)-2=(C, C)-\left(C,-K_{X}\right) .
$$

Since $C$ is irreducible, we have $p_{a}(C) \geq 0$. All this forces $p_{a}(C)=0$ and $(C, C)=\left(C, K_{X}\right)=$ -1 , and thus $C$ is exceptional.

Proof of Theorem 1.6. Let $f: X \rightarrow Y$ be a birational $k$-morphism with $Y$ minimal, and write

$$
X=X_{0} \rightarrow X_{1} \rightarrow \cdots \rightarrow X_{r}=Y
$$

for a factorization of $f$ as in Proposition 1.7. By Proposition 1.8 we need only consider the following cases:

(1) $Y=\mathbb{P}_{k}^{2}$. We claim that no point that is blown-up in one step of the factorization (2) may lie on the exceptional divisor of a previous blow-up: otherwise $X_{\bar{k}}$ would contain a curve with self-intersection less than -1 , contradicting Lemma 1.9. Hence $X$ is the blow-up of $\mathbb{P}_{k}^{2}$ at $r$ distinct closed $k$-points. We conclude that $d=K_{X}^{2}=9-r$, as claimed; note that $d=\left(K_{X}, K_{X}\right) \geq 1$ since $-K_{X}$ is ample, and so $0 \leq r \leq 8$. Suppose that 3 of these points lie on a line $L$. Let $f_{\bar{k}}^{-1} L_{\bar{k}}$ denote the strict transform of $L_{\bar{k}}$ for the base-extension $f_{\bar{k}}: X_{\bar{k}} \rightarrow Y_{\bar{k}}$. Then $\left(f_{\bar{k}}^{-1} L_{\bar{k}}, f_{\bar{k}}^{-1} L_{\bar{k}}\right)<-1$, but this is impossible by Lemma 1.9. Similarly, if 6 of the blown-up points lie on a conic $Q$, or if 8 points lie on a singular cubic $C$ with one of the points at the singularity, then $\left(f_{\bar{k}}^{-1} Q_{\bar{k}}, f_{\bar{k}}^{-1} Q_{\bar{k}}\right)<-1$, or $\left(f_{\bar{k}}^{-1} C_{\bar{k}}, f_{\bar{k}}^{-1} C_{\bar{k}}\right)<-1$, respectively, which is not possible. Hence the blown-up points are in general position.

(2) $Y=\mathbb{P}_{k}^{1} \times \mathbb{P}_{k}^{1}$. If $X=Y$ then $X$ is a del Pezzo surface of degree 8. Otherwise, we may contract the two nonintersecting $(-1)$-curves of $X_{r-1}$ and obtain a birational morphism $\phi: X_{r-1} \rightarrow \mathbb{P}_{k}^{2}$. We may use the map $\phi$ to construct a new birational morphism $X \rightarrow \mathbb{P}_{k}^{2}$, given by

$$
X=X_{0} \rightarrow X_{1} \rightarrow \cdots \rightarrow X_{r-1} \stackrel{\phi}{\rightarrow} \mathbb{P}_{k}^{2},
$$

and thus we may reduce this case to the previous case.

(3) $Y=\mathbb{F}_{n}, n \geq 2$. There is a curve $C \subseteq\left(\mathbb{F}_{n}\right)_{\bar{k}}$ whose divisor class satisfies $(C, C)<-1$. Let $f_{\bar{k}}^{-1}(C)$ denote the strict transform of $C$ in $X_{\bar{k}}$ for the base-extension $f_{\bar{k}}: X_{\bar{k}} \rightarrow$ $\left(\mathbb{F}_{n}\right)_{\bar{k}}$. Then $\left(f_{\bar{k}}^{-1} C, f_{\bar{k}}^{-1} C\right)<-1$, but this is impossible by Lemma 1.9.

1.5. Further properties of del Pezzo surfaces. The basic references on the subject are [Man74], [Dem80] and [Kol96, III.3].

1.5.1. The Picard group. Let $X$ be a del Pezzo surface over a field $k$ of degree $d$. Recall that an exceptional curve on $X$ is an irreducible curve $C$ on $X_{\bar{k}}$ such that $(C, C)=\left(C, K_{X}\right)=-1$. Theorem 1.6 shows that exceptional curves on $X$ are already defined over $k^{s}$.

We have seen that if $X_{k^{s}} ¥ \mathbb{P}_{k^{s}}^{1} \times \mathbb{P}_{k^{s}}^{1}$ then $X_{k^{s}}$ is isomorphic to a blow-up of $\mathbb{P}_{k^{s}}^{2}$ at $r:=9-d$ closed points $\left\{P_{1} \ldots, P_{r}\right\}$ in general position. It follows that the group $\operatorname{Pic} X_{k^{s}}$ is isomorphic to $\mathbb{Z}^{10-d}$ (see [Har77, Proposition V.3.2]); if $d \leq 7$ then it is generated by the classes of exceptional curves. Let $e_{i}$ be the class of an exceptional curve corresponding to $P_{i}$ 
under the blow-up map, and let $\ell$ be the class of the pullback of a line in $\mathbb{P}_{k^{s}}^{2}$ not passing through any of the $P_{i}$. Then $\left\{e_{1}, \ldots, e_{r}, \ell\right\}$ is a basis for Pic $X_{k^{s}}$. Note that

$$
\left(e_{i}, e_{j}\right)=-\delta_{i j}, \quad\left(e_{i}, \ell\right)=0, \quad(\ell, \ell)=1,
$$

where $\delta_{i j}$ is the usual Kronecker delta function. With respect to this basis, the anticanonical class is given by $-K_{X}=3 \ell-\sum e_{i}$.

This basis also allows us to interpret the exceptional curves in terms of strict transforms of our blow-up: for example, if $C=\ell-e_{1}-e_{2}$ as classes in Pic $X_{k^{s}}$, then $C$ is the strict transform of the line in $\mathbb{P}^{2}$ through the points $P_{1}$ and $P_{2}$.

The number of exceptional curves on $X$ is finite, and is computed as follows: if $C=$ $a \ell-\sum_{i=1}^{r} b_{i} e_{i}$ is an exceptional curve, then

$$
\begin{aligned}
& a^{2}-\sum_{i=1}^{r} b_{i}^{2}=-1 . \\
& 3 a-\sum_{i=1}^{r} b_{i}=1 .
\end{aligned}
$$

These equations are easily solved (keep in mind that $a$ and $b_{i}$ are integers). The number of exceptional curves on $X$ as $r$ (and hence $d$ ) varies is shown in Table 1 .

\begin{tabular}{|c|ccccccc|}
\hline$d(X)$ & 7 & 6 & 5 & 4 & 3 & 2 & 1 \\
\hline \# of exceptional curves & 3 & 6 & 10 & 16 & 27 & 56 & 240 \\
\hline
\end{tabular}

TABLE 1. Number of exceptional curves on $X$

1.5.2. Root systems. Suppose that $r=9-d \geq 3$. The orthogonal complement $K_{X}^{\perp}$ of $K_{X}$ in Pic $X_{k^{s}} \otimes \mathbb{R}$, equipped with the negative of the intersection form on $X$, is a Euclidean space. Its group of orthogonal transformations $O\left(K_{X}^{\perp}\right)$, being an intersection of a compact group and a discrete group, is finite. The set of vectors

$$
R_{r}:=\left\{v \in \operatorname{Pic} X_{k^{s}}:\left(v, K_{X}\right)=0,(v, v)=-2\right\}
$$

is a root system of rank $r$. Using standard facts about root systems, it is not too hard to identify $R_{r}$ as $r$ varies; see [Man74, Theorem 25.4]. An important fact for our purposes is that the group of automorphisms of Pic $X_{k^{s}}$ that preserve the intersection form and $K_{X}$ coincides with the Weyl group $W\left(R_{r}\right)$ of the root system $R_{r}$ [Man74, Theorem 23.9], i.e., $O\left(K_{X}\right)^{\perp}=W\left(R_{r}\right)$.

\begin{tabular}{|c|cccccc|}
\hline$r$ & 3 & 4 & 5 & 6 & 7 & 8 \\
\hline$R_{r}$ & $A_{1} \times A_{2}$ & $A_{4}$ & $D_{5}$ & $E_{6}$ & $E_{7}$ & $E_{8}$ \\
\hline
\end{tabular}

TABLE 2. Root systems on Pic $X_{k^{s}}$ 
1.5.3. Galois action on the Picard group. The Galois group Gal $\left(k^{s} / k\right)$ acts on Pic $X_{k^{s}}$ as follows. For $\sigma \in \operatorname{Gal}\left(k^{s} / k\right)$, let $\tilde{\sigma}: \operatorname{Spec} k^{s} \rightarrow \operatorname{Spec} k^{s}$ be the corresponding morphism. Then

$$
\operatorname{id}_{X} \times \tilde{\sigma}: X_{k^{s}} \rightarrow X_{k^{s}}
$$

induces an automorphism $\left(\operatorname{id}_{X} \times \tilde{\sigma}\right)^{*}$ of $\operatorname{Pic} X_{k^{s}}$. This gives a group homomorphism

$$
\operatorname{Gal}\left(k^{s} / k\right) \rightarrow \operatorname{Aut}\left(\operatorname{Pic} X_{k^{s}}\right) \quad \sigma \mapsto\left(\operatorname{id}_{X} \times \tilde{\sigma}\right)^{*} .
$$

The action of $\operatorname{Gal}\left(k^{s} / k\right)$ on $\operatorname{Pic}\left(X_{k^{s}}\right)$ fixes the canonical class $K_{X}$ and preserves the intersection pairing; in particular, the action of $\operatorname{Gal}\left(k^{s} / k\right)$ takes exceptional curves to exceptional curves (see [Man74, Theorem 23.8]). By our discussion in $\S 1.5 .2$, it follows that $\operatorname{Gal}\left(k^{s} / k\right)$ acts as a subgroup of $W\left(R_{r}\right)$ on $\operatorname{Pic} X_{k^{s}}$.

Let $K$ be the smallest extension of $k$ in $k^{s}$ over which all exceptional curves of $X$ are defined. We say that $K$ is the splitting field of $X$. The natural action of $\operatorname{Gal}\left(k^{s} / k\right)$ on $\operatorname{Pic} X_{k^{s}} \cong \operatorname{Pic} X_{K}$ factors through the quotient $\operatorname{Gal}(K / k)$, giving a homomorphism

$$
\phi_{X}: \operatorname{Gal}(K / k) \rightarrow \operatorname{Aut}\left(\operatorname{Pic} X_{K}\right) .
$$

If we have equations with coefficients in $K$ for an exceptional curve $C$ of $X$, then an element $\sigma \in \operatorname{Gal}(K / k)$ acts on $C$ by applying $\sigma$ to each coefficient.

The map (3) conjecturally determines much of the arithmetic of $X$ : there is a conjecture of Colliot-Thélène and Sansuc (first asked as a question in [CTS80], based on evidence published in [CTCS80, CTS82]) that says that the Brauer-Manin obstruction explains all violations of the Hasse principle for del Pezzo surfaces over a number field $k$. One can use $\phi_{X}$ to compute the Brauer-Manin obstruction, so if the conjecture is true, then one can decide whether $X$ has a $k$-point.

1.5.4. Anticanonical models. For any scheme $X$ and line sheaf $\mathscr{L}$ on $X$, we may construct the graded ring

$$
R(X, \mathscr{L}):=\bigoplus_{m \geq 0} H^{0}\left(X, \mathscr{L}^{\otimes m}\right) .
$$

When $\mathscr{L}=\omega_{X}^{\otimes-1}$, we call $R\left(X, \omega_{X}^{\otimes-1}\right)$ the anticanonical ring of $X$. If $X$ is a del Pezzo surface then $X$ is isomorphic to the scheme Proj $R\left(X, \omega_{X}^{\otimes-1}\right)$, because $\omega_{X}^{\otimes-1}$ is ample. This scheme is known as the anticanonical model of the del Pezzo surface.

The construction of anticanonical models is reminiscent of the procedure that yields a Weierstrass model of an elliptic curve. In fact, we can use the Riemann-Roch theorem for surfaces (and Kodaira vanishing-which is valid even in positive characteristic for rational surfaces) to prove the following dimension formula for a del Pezzo surface $X$ over $k$ of degree $d$ :

$$
h^{0}\left(X,-m K_{X}\right)=\frac{m(m+1)}{2} d+1 ;
$$

see [Kol96, Corollary III.3.2.5] or [CO99]. For example, if $X$ has degree 1 , then the anticanonical model for $X$ is a smooth sextic hypersurface in $\mathbb{P}_{k}(1,1,2,3)$, and we may compute such a model, up to isomorphism, as follows:

(1) Choose a basis $\{x, y\}$ for the 2-dimensional $k$-vector space $\mathrm{H}^{0}\left(X,-K_{X}\right)$.

(2) The elements $x^{2}, x y, y^{2}$ of $\mathrm{H}^{0}\left(X,-2 K_{X}\right)$ are linearly independent. However, $h^{0}\left(X,-2 K_{X}\right)=4$; choose an element $z$ to get a basis $\left\{x^{2}, x y, y^{2}, z\right\}$ for this $k$-vector space. 
(3) The elements $x^{3}, x^{2} y, x y^{2}, y^{3}, x z, y z$ of $\mathrm{H}^{0}\left(X,-3 K_{X}\right)$ are linearly independent, but $h^{0}\left(X,-3 K_{X}\right)=7$. Choose an element $w$ to get a basis $\left\{x^{3}, x^{2} y, x y^{2}, y^{3}, x z, y z, w\right\}$ for this $k$-vector space.

(4) The vector space $\mathrm{H}^{0}\left(X,-6 K_{X}\right)$ is 22-dimensional, so the 23 elements

$$
\begin{aligned}
& \left\{x^{6}, x^{5} y, x^{4} y^{2}, x^{3} y^{3}, x^{2} y^{4}, x y^{5}, y^{6}, x^{4} z, x^{3} y z, x^{2} y^{2} z, x y^{3} z,\right. \\
& \left.\quad y^{4} z, x^{2} z^{2}, x y z^{2}, y^{2} z^{2}, z^{3}, x^{3} w, x^{2} y w, x y^{2} w, y^{3} w, x z w, y z w, w^{2}\right\}
\end{aligned}
$$

must be $k$-linearly dependent. Let $f(x, y, z, w)=0$ be a linear dependence relation among these elements. Then an anticanonical model of $X$ is $\operatorname{Proj} k[x, y, z, w] /(f)$, where $x, y, z, w$ are variables with weights $1,1,2$ and 3 respectively. This way $X$ may be described as the (smooth) sextic hypersurface $V(f)$ in $\mathbb{P}_{k}(1,1,2,3)$.

For more details on this construction, see [CO99, pp.1199-1201].

Remark 1.10. If $k$ is a field of characteristic not equal to 2 or 3 , then in step (4) above we may complete the square with respect to the variable $w$ and the cube with respect to the variable $z$ to obtain an equation $f(x, y, z, w)=0$ involving only the monomials

$$
\left\{x^{6}, x^{5} y, x^{4} y^{2}, x^{3} y^{3}, x^{2} y^{4}, x y^{5}, y^{6}, x^{4} z, x^{3} y z, x^{2} y^{2} z, x y^{3} z, y^{4} z, z^{3}, w^{2}\right\} .
$$

Moreover, we may also rescale the variables so that the coefficients of $w^{2}$ and $z^{3}$ are \pm 1 .

Remark 1.11. If $X$ has degree $d \geq 3$, then the anticanonical model recovers the usual description of $X$ as a smooth degree $d$ surface in $\mathbb{P}_{k}^{d}$. In particular, when $d=3$ we get a smooth cubic surface in $\mathbb{P}_{k}^{3}$. If $X$ has degree 2 then the anticanonical model is a smooth quartic hypersurface in the weighted projective space $\mathbb{P}_{k}(1,1,1,2)$; such a surface can then be thought of as a double cover of a $\mathbb{P}_{k}^{2}$ ramified along a quartic curve.

Remark 1.12. If we write a del Pezzo surface $X$ of degree 1 over a field $k$ as the smooth sextic hypersurface $V(f(x, y, z, w))$ in $\mathbb{P}_{k}(1,1,2,3)$, then $\{x, y\}$ is a basis for $\mathrm{H}^{0}\left(X,-K_{X}\right)$. In particular, $\left|-K_{X}\right|$ has a unique base point: $[0: 0: 1: 1]$.

\section{Lecture 2: Arithmetic of Del Pezzo surfaces of Degree at least 5}

The goal for this lecture is to prove the following theorem.

Theorem 2.1. Let $X$ be a del Pezzo surface of degree $d \geq 5$. If $X(k) \neq \emptyset$, then $X$ is $k$-birational to $\mathbb{P}_{k}^{2}$. This hypothesis is automatically satisfied if $d=5$ or 7 .

In particular, if $k$ is a global field then $X$ satisfies weak approximation. In addition, $X(k) \neq \emptyset$ provided $X\left(k_{v}\right) \neq \emptyset$ for all $v \in \Omega_{k}$ (i.e., del Pezzo surfaces of degree at least 5 satisfy the Hasse principle).

We will prove this theorem by a case-by-case analysis on the degree of $X$, making attributions as we go along. Our exposition is influenced by [CT99, §4].

2.1. Case 1: $d(X)=9$. By Theorem 1.6, $X_{\bar{k}}$ is isomorphic to $\mathbb{P}_{\bar{k}}^{2}$, i.e., $X_{\bar{k}}$ is a form of the projective plane. It is a classical theorem of Châtelet that such a surface is $k$-isomorphic to $\mathbb{P}_{k}^{2}$ if and only if $X(k) \neq \emptyset$. We will prove this result more generally for Severi-Brauer varieties. 
Definition 2.2. A Severi-Brauer variety is a projective scheme $X$ over a field $k$ that becomes isomorphic to some $n$-dimensional projective space upon passage to an algebraic closure $\bar{k}$ of $k$, i.e., $X_{\bar{k}} \cong \mathbb{P}_{\bar{k}}^{n}$ for some $n$.

Theorem 2.3 (Châtelet). Let $X$ be a Severi-Brauer variety of dimension $n$ over a field $k$. The following are equivalent:

(1) $X$ is $k$-isomorphic to $\mathbb{P}_{k}^{n}$;

(2) $X(k) \neq \emptyset$.

Proof. The proof we give here is due to Endre Szabó; we follow the exposition in [GS06]. The implication $(1) \Longrightarrow(2)$ is clear. We claim that if $X(k) \neq \emptyset$ then $X$ contains a twisted linear subvariety $D$ of codimension 1 . Let $\pi: Y \rightarrow X$ be the blow-up of $X$ at a $k$-rational point $P$. The variety $Y_{\bar{k}}$ is isomorphic to the blow-up of $\mathbb{P}_{\bar{k}}^{n}$ at a closed point and we can think of it as a subvariety of $\mathbb{P}_{\bar{k}}^{n} \times \mathbb{P}_{\bar{k}}^{n-1}$. Let $\psi_{\bar{k}}: Y_{\bar{k}} \rightarrow \mathbb{P}_{\bar{k}}^{n-1}$ be the projection onto the second factor. Choose a hyperplane $L$ on the exceptional divisor $E \cong \mathbb{P}_{k}^{n-1}$ of the blow-up. The subvariety $D_{\bar{k}}:=\pi_{\bar{k}}\left(\psi_{\bar{k}}^{-1}\left(\psi_{\bar{k}}\left(L_{\bar{k}}\right)\right)\right)$ of $X_{\bar{k}}$ is a hyperplane in $\mathbb{P}_{\bar{k}}^{n}$.

Choose an ample divisor $A$ of $X$, of degree $d$ over $\bar{k}$. The linear system $\left|\pi^{*} A-d E\right|$ defines a $k$-rational map $\phi: Y \rightarrow \mathbb{P}_{k}^{N}$. Since $\left(\pi^{*} A-d E\right)_{\bar{k}}$ has degree 0 on the fibers of $\psi_{\bar{k}}$ and degree $d$ on $E_{\bar{k}}$, the map $\phi_{\bar{k}}$ factors as $\psi_{\bar{k}}$ followed by the $d$-uple embedding. In particular, $\phi$ is defined everywhere. The subvariety $D:=\pi\left(\phi^{-1}(\phi(L))\right)$ of $X$ is defined over $k$ and becomes $D_{\bar{k}}$ after base extension to $\bar{k}$.

The linear system $|D|$ gives a rational map $\phi_{D}: X \rightarrow \mathbb{P}_{k}^{n_{1}}$. Over $\bar{k}, D$ becomes a hyperplane and thus $\left(\phi_{D}\right)_{\bar{k}}$ is an isomorphism with $\mathbb{P}_{\bar{k}}^{n}$. This shows that $n_{1}=n$, and $\phi$ is an everywhere-defined isomorphism.

Over a global field, forms of $\mathbb{P}_{k}^{n}$ satisfy the Hasse principle. This also follows from work of Châtelet [Châ44].

Theorem 2.4 (Châtelet). Severi-Brauer varieties over a global field satisfy the Hasse principle.

Idea of the proof. Let $S B_{n}(k)$ be the pointed set of isomorphism classes of Severi-Brauer varieties of dimension $n$ over $k$ (the base point is the class of $\mathbb{P}_{k}^{n}$ ). There is a base-point preserving bijection

$$
S B_{n}(k) \longleftrightarrow \mathrm{H}^{1}\left(\operatorname{Gal}\left(k^{s} / k\right), \mathrm{PGL}_{n+1}\left(k^{s}\right)\right) ;
$$

see [GS06, Theorem 5.2.1]. If $K$ is a finite Galois extension of $k$, then there is a base-point preserving bijection between $k$-isomorphism classes of central simple algebras of degree $n$ split by $K$ and $\mathrm{H}^{1}\left(\mathrm{Gal}(K / k), \mathrm{PGL}_{n+1}(K)\right)$; see [GS06, Theorem 2.4.3]. Thus, to a SeveriBrauer variety $X$ over $k$, we may naturally associate a class of $\operatorname{Br}(k)$ in a unique way, and this class is trivial if and only if $X \cong \mathbb{P}_{k}^{n}$, i.e., if and only if $X(k) \neq \emptyset$, by Theorem 2.3. The theorem now follows from the fact that the map

$$
\operatorname{Br}(k) \rightarrow \bigoplus_{v \in \Omega_{k}} \operatorname{Br}\left(k_{v}\right), \quad A \mapsto\left(A \otimes_{k} k_{v}\right)
$$

is an injection [NSW08, Theorem 8.1.17]. 
2.2. Case 2: $d(X)=8$. By Theorem 1.6, $X_{k^{s}}$ is isomorphic to either a blow-up of $\mathbb{P}_{k^{s}}^{2}$ at a point or to $\mathbb{P}_{k^{s}}^{1} \times \mathbb{P}_{k^{s}}^{1}$. We deal with these cases separately. In the former case, there is exactly one exceptional curve on $X$, and $\operatorname{Gal}\left(k^{s} / k\right)$ must fix it (see 1.5.3). Blowing down this curve we obtain a del Pezzo surface of degree 9 with a $k$-point, so by Theorem 2.3 we conclude that $X$ is $k$-birational to $\mathbb{P}_{k}^{2}$. In particular, $X(k) \neq \emptyset$, so these surfaces trivially satisfy the Hasse principle.

Suppose now that $X_{k^{s}}$ is isomorphic to $\mathbb{P}_{k^{s}}^{1} \times \mathbb{P}_{k^{s}}^{1}$. Then Pic $X_{k^{s}} \cong \mathbb{Z} L_{1} \oplus \mathbb{Z} L_{2}$, where $L_{1}$ and $L_{2}$ give the two rulings of $\mathbb{P}_{k^{s}}^{1} \times \mathbb{P}_{k^{s}}^{1}$. Note that $K_{X}=-2\left(L_{1}+L_{2}\right)$, and hence $L_{1}+L_{2}$ is stable under the action of $\operatorname{Gal}\left(k^{s} / k\right)$. If $X(k) \neq \emptyset$, or if $k$ is a global field and $X\left(k_{v}\right) \neq \emptyset$ for all $v \in \Omega_{k}$, then the inclusion

$$
\operatorname{Pic} X \rightarrow\left(\operatorname{Pic} X_{k^{s}}\right)^{\operatorname{Gal}\left(k^{s} / k\right)}
$$

is an isomorphism (see $§ 3.4)$. In either case, the class of $L_{1}+L_{2}$ may be represented over $k$ by some divisor $D$. The linear system $|D|$ gives an embedding $\phi_{D}: X \rightarrow \mathbb{P}_{k}^{3}$ of $X$ as a quadric surface, defined over $k$. If $P \in X(k)$, then composing $\phi_{D}$ with projection away from $\phi_{D}(P)$ we obtain a $k$-birational map $X \rightarrow \mathbb{P}_{k}^{2}$.

If $k$ is a global field and $X\left(k_{v}\right) \neq \emptyset$ for all $v \in \Omega_{k}$, then $\left(\phi_{D}\right)_{k_{v}}\left(X_{k_{v}}\right)$ is a quadric in $\mathbb{P}_{k_{v}}^{3}$, and $\phi_{D}(X)\left(k_{v}\right) \neq \emptyset$ for all $v \in \Omega_{k}$. Quadrics over global fields satisfy the Hasse principle, so $\phi_{D}(X)(k) \neq \emptyset$ and hence $X(k) \neq \emptyset$.

2.3. Case 3: $d(X)=7$. By Theorem 1.6, $X_{k^{s}}$ is isomorphic to a blow-up of $\mathbb{P}_{k^{s}}^{2}$ at two points. There are only three exceptional curves on $X$, and their configuration is quite simple. Label these curves $e_{1}, e_{2}$ and $e_{3}$; without loss of generality we have

$$
\left(e_{1}, e_{2}\right)=\left(e_{2}, e_{3}\right)=1 \text { and }\left(e_{1}, e_{3}\right)=0 .
$$

Since the action of $\operatorname{Gal}\left(k^{s} / k\right)$ on $\operatorname{Pic} X_{k^{s}}$ respects the intersection pairing, it follows that $e_{2}$ is defined over $k$. Contracting it we obtain a del Pezzo surface of degree 8 with a point, and hence $X(k) \neq \emptyset$ by the Lang-Nishimura lemma. In particular, del Pezzo surfaces of degree 7 trivially satisfy the Hasse principle. It follows from our work above that $X$ is $k$-birational to $\mathbb{P}_{k}^{2}$.

2.4. Case 4: $d(X)=6$. By Theorem 1.6, $X_{k^{s}}$ is isomorphic to a blow-up of $\mathbb{P}_{k^{s}}^{2}$ at three non-colinear points. If we have a point $P \in X(k)$ that lies on at least one exceptional curve then one can show that $X$ is not minimal and conclude using our work above. If $P$ is not on any exceptional curve, then we may blow-up $P$ to obtain a del Pezzo surface of degree 5. On this surface, there are three exceptional curves that meet the exceptional divisor of the blow-up, are pairwise skew, and form a $\operatorname{Gal}\left(k^{s} / k\right)$-set. Contracting them we obtain a del Pezzo surface of degree 8 that contains a rational point. Hence $X$ is $k$-birational to $\mathbb{P}_{k}^{2}$.

The exceptional curves of $X_{k^{s}}$ form a "hexagon." Let $e_{1}, \ldots, e_{6}$ be the exceptional curves of $X$, numbered to correspond clockwise to the vertices of the hexagon. Thus $\left\{e_{1}, e_{3}, e_{5}\right\}$ and $\left\{e_{2}, e_{4}, e_{6}\right\}$ are triplets of curves that do not pairwise intersect, and $\left\{e_{1}, e_{4}\right\},\left\{e_{2}, e_{5}\right\}$ and $\left\{e_{3}, e_{6}\right\}$ are couples of curves whose vertices in the hexagon are "as far apart as possible." Since the action of $\operatorname{Gal}\left(k^{s} / k\right)$ respects the intersection form, it induces an action on the sets

$$
T:=\left\{\left\{e_{1}, e_{3}, e_{5}\right\},\left\{e_{2}, e_{4}, e_{6}\right\}\right\} \quad \text { and } D:=\left\{\left\{e_{1}, e_{4}\right\},\left\{e_{2}, e_{5}\right\},\left\{e_{3}, e_{6}\right\}\right\} .
$$

If any element of $T$ or $D$ is fixed by the action of $\operatorname{Gal}\left(k^{s} / k\right)$, then it is defined over $k$. Hence there exist 
- an element of $T$ defined over a field extension $K$ of $k$ with $[K: k] \mid 2$.

- an element of $D$ defined over a field extension $L$ of $k$ with $[L: k] \mid 3$.

On $X_{K}$ we may contract the curves in the fixed element of $T$ to obtain a del Pezzo surface of degree 9. On $X_{L}$ we may contract the curves in the fixed element of $D$ to obtain a del Pezzo surface of degree 8 that is a form of $\mathbb{P}_{L}^{1} \times \mathbb{P}_{L}^{1}$.

If $k$ is a global field and $X\left(k_{v}\right) \neq \emptyset$ for all $v \in \Omega_{v}$, then both $X_{L}$ and $X_{K}$ also have points everywhere locally. Since del Pezzo surfaces of degree 8 and 9 satisfy the Hasse principle, it follows that $X_{K}$ is $K$-birational to $\mathbb{P}_{K}^{2}$ and $X_{L}$ is $L$-birational to $\mathbb{P}_{L}^{2}$. This means that there is a closed point $P_{1}$ of $X$ consisting of a pair of $K$-points that are conjugate, as well as a closed point $P_{2}$ of $X$ consisting of three $L$-points that are conjugate. Choose a four-dimensional linear subspace $L$ of $\mathbb{P}_{k}^{4}$ that passes through $P_{1}$ and $P_{2}$. By Bezout's theorem, $X_{\bar{k}} \cap L_{\bar{k}}$ consists of 6 points, counted with multiplicity (because $X$ has degree 6 - this assumes the intersection is the right dimension; when the intersection contains a curve one has to do a little more work). The points $P_{1}$ and $P_{2}$ account for 5 of the geometric points in $X_{\bar{k}} \cap L_{\bar{k}}$. The remaining point must be defined over $k$ (how else would Galois act on it?). Thus del Pezzo surfaces of degree 6 satisfy the Hasse principle (this proof is essentially due to Colliot-Thélène [CT72]).

2.5. Case 5: $d(X)=5$. We shall assume first that $X(k) \neq \emptyset$. If $X$ contains a $k$-point $P$ that does not lie on any exceptional curve, then the blow-up of $X$ at $P$ is a del Pezzo surface $Y$ of degree 4. Let $e$ be the exceptional curve of $Y$ corresponding to the blown-up point $P$. Examining the graph of intersections of exceptional curves on $Y$, we see that there are five curves that do not intersect $e$, and that are pairwise skew to each other. These five curves form an invariant set under the action of $\operatorname{Gal}\left(k^{s} / k\right)$ because $e$ is defined over $k$. Contracting this set we obtain a del Pezzo surface of degree 9 with $k$-point (since $X(k) \neq \emptyset$ ), so by Theorem 2.3 we conclude that $X$ is $k$-birational to $\mathbb{P}_{k}^{2}$. An easy case-by-case analysis shows that if $P$ lies on at least one exceptional curve, then it is possible to contract at least one exceptional curve over $k$ and thus reduce to previous cases to conclude that $X$ is $k$-birational to $\mathbb{P}_{k}^{2}$.

Theorem 2.5 (Enriques, Swinnerton-Dyer, Skorobogatov, Shepherd-Barron, Kollár). Let $X$ be a del Pezzo surface of degree 5 over a field $k$. Then $X(k) \neq \emptyset$. In particular, if $k$ is a global field, then del Pezzo surfaces of degree 5 trivially satisfy the Hasse principle.

Sketch of proof. We follow Swinnerton-Dyer's approach [SD72]; other proofs can be found in [Enr97, Sko93, Kol96, SB92, Has09]. Recall that the anticanonical model of $X$ is a quintic surface in $\mathbb{P}_{k}^{5}$ (i.e., the linear system $\left|-K_{X}\right|$ embeds $X$ as a quintic in $\mathbb{P}_{k}^{5}$; see $\S 1.5 .4$ ). Under this embedding, $X$ is cut out by 5 quadrics, defined over $k$. It suffices to verify this claim after a base-extension of the field. Over $k^{s}$, the surface $X_{k^{s}}$ is isomorphic to the blow-up of $\mathbb{P}_{k^{s}}^{2}$ at four points, no three of which are colinear. By a projective transformation (defined over $k^{s}$ ), we may assume that these points are

$$
[1: 0: 0],[0: 1: 0],[0: 0: 1] \text { and }[1: 1: 1] \text {. }
$$

It is now not difficult to check that the ideal $I\left(X_{k^{s}}\right)$ of $X_{k^{s}} \subseteq \mathbb{P}_{k^{s}}^{5}$ is generated by 5 quadrics. Let $Q$ be a general element of $I\left(X_{k^{s}}\right)_{2}$, let $\Pi_{1}$ be a general plane lying in $Q$, and let $L$ be a general threefold through $\Pi_{1}$ of $X$. Then

$$
Q \cdot L=\Pi_{11}+\Pi_{2}
$$


where $\Pi_{2}$ is another plane residual to $\Pi_{1}$. One can show that

$$
X \cdot L=\left(X \cdot \Pi_{1}\right)_{Q}+\left(X \cdot \Pi_{1}\right)_{Q},
$$

and that, after possibly interchanging $\Pi_{1}$ and $\Pi_{2}$,

$$
\operatorname{deg}\left(X \cdot \Pi_{1}\right)_{Q}=2 \text { and } \operatorname{deg}\left(X \cdot \Pi_{2}\right)_{Q}=3
$$

These two equalities can now be used to show that $\Pi_{i}$ may be chosen in such a way that it is defined over an extension $K$ of $k$ with $[K: k]=2^{n}$ with $0 \leq n \leq 3$. Suppose for simplicity that $n=1$. Let $P$ be a $K$-point of $\Pi_{1}$ and let $P^{c}$ be its conjugate over $k$. Let $H \subset \mathbb{P}_{k}^{5}$ be any hyperplane defined over $K$. Then $H_{k^{s}} \cdot X_{k^{s}}$ is a curve $C$ of genus one defined over $K$ containing the zero-cycle $P+P^{c}$, and a zero-cycle of degree 5 obtained by intersecting $X$ with any 3 -dimensional linear subspace of $H$. Hence $C$ contains a zero-cycle of degree 1 and thus a $k$-rational point. If $n>1$, we just repeat this procedure a total of $n$ times.

Remark 2.6. Here is an alternative strategy [Has09, Exercise 3.1.4]: show that if $Q_{1}, Q_{2}, Q_{3}$ are general elements of $I(X)_{2}$ then

$$
V\left(Q_{1}, Q_{2}, Q_{3}\right)=X \cup W,
$$

where $W_{k^{s}}$ is isomorphic to the blow-up of $\mathbb{P}_{k^{s}}^{2}$ at a point. By our work in $\S 2.2$, the exceptional divisor of $W_{k^{s}}$ is defined over $k$. Now show that this exceptional divisor intersects $X$ in one point.

Proof of Theorem 2.1. At this point, it only remains to note that since $X$ is $k$-birational to $\mathbb{P}_{k}^{2}$, it must satisfy weak approximation, by Lemma 1.2 .

\section{Lecture 3: Counterexamples in Small Degree}

We have seen that del Pezzo surfaces of degree at least 5 over global fields satisfy both the Hasse principle and weak approximation. This is no longer the case for surfaces of lower degree. Of course, a counterexample to the Hasse principle immediately gives a counterexample to weak approximation. However, in degrees 2, 3 or 4, there exist examples of surfaces with a Zariski dense set of points for which weak approximation fails. Curiously, del Pezzo surfaces of degree 1 always have a $k$-rational point: the unique base-point of the anticanonical linear system (see Remark 1.12). These surfaces, however, need not satisfy weak approximation.

In Table 3 we have compiled a list of references for the earliest recorded counterexamples to the Hasse principle and weak approximation in low degrees. A little care must be taken with counterexamples to weak approximation: for example, by Lemma 1.2, if $X$ is a counterexample to weak approximation and $X(k) \neq \emptyset$, then the blow-up of $X$ at a $k$-point is also a counterexample to weak approximation. Thus, when dealing with counterexamples to weak approximation on del Pezzo surfaces, it is important to make sure that the surfaces are $k$-minimal: the surface should not have a Galois-stable set of pairwise skew exceptional curves.

3.1. The Brauer-Manin set I. Let $X$ be a nice variety over a global field $k$. Since $X$ is proper, we have $\prod_{v} X\left(k_{v}\right)=X\left(\mathbf{A}_{k}\right)$. In [Man71], Manin used the Brauer group of the variety to construct an intermediate "obstruction set" between $X(k)$ and $X\left(\mathbf{A}_{k}\right)$ :

$$
X(k) \subseteq X\left(\mathbf{A}_{k}\right)^{\mathrm{Br}} \subseteq X\left(\mathbf{A}_{k}\right) .
$$




\begin{tabular}{|c|ccccc|}
\hline Phenomenon & $d \geq 5$ & $d=4$ & $d=3$ & $d=2$ & $d=1$ \\
\hline Hasse principle & $\checkmark$ & [BSD75] & {$[$ SD62] } & {$[$ KT04] } & $\checkmark$ \\
Weak approximation & $\checkmark$ & [CTS77] & [SD62] & [KT08] & [VA08] \\
\hline
\end{tabular}

TABle 3. Arithmetic phenomena on del Pezzo surfaces over global fields. A check mark $(\checkmark)$ indicates that the relevant arithmetic phenomenon holds for the indicated class of surfaces. An entry with a reference indicates the existence of a counterexample to the arithmetic phenomenon which can be found in the paper cited.

In fact, the set $X\left(\mathbf{A}_{k}\right)^{\mathrm{Br}}$ already contains the closure of $X(k)$ for the adelic topology and thus may be used to explain the failure of both the Hasse principle and weak approximation on many classes of varieties.

Definition 3.1. Let $X$ be a nice $k$-variety, and assume that $X\left(\mathbf{A}_{k}\right) \neq \emptyset$. We say that $X$ is a counter-example to the Hasse principle explained by the Brauer-Manin obstruction if $X\left(\mathbf{A}_{k}\right)^{\mathrm{Br}}=\emptyset$. We say that $X$ is a counter-example to the weak approximation explained by the Brauer-Manin obstruction if $X\left(\mathbf{A}_{k}\right) \backslash X\left(\mathbf{A}_{k}\right)^{\mathrm{Br}} \neq \emptyset$.

3.2. Brauer groups of schemes. Recall that the Brauer group of a field $k$ can be defined in two different ways: as the set of similarity classes of central simple algebras over $k$, or as the Galois cohomology group $\mathrm{H}^{2}\left(\operatorname{Gal}\left(k^{s} / k\right), k^{s *}\right)$. These two points of view can be naturally identified, and each has its own advantages, depending on context. Both definitions have natural generalizations to schemes, but these generalizations need no longer be naturally isomorphic.

Definition 3.2. An Azumaya algebra on a scheme $X$ is an $\mathscr{O}_{X}$-algebra $\mathcal{A}$ that is coherent and locally free as an $\mathscr{O}_{X}$-module, such that the fiber $\mathcal{A}(x):=\mathcal{A} \otimes_{\mathscr{O}_{X, x}} k(x)$ is a central simple algebra over the residue field $k(x)$ for each $x \in X$.

Two Azumaya algebras $\mathcal{A}$ and $\mathcal{B}$ on $X$ are similar if there exist nonzero locally free coherent $\mathscr{O}_{X}$-modules $\mathcal{E}$ and $\mathcal{F}$ such that

$$
\mathcal{A} \otimes_{\mathscr{O}_{X}} \operatorname{End}_{\mathscr{O}_{X}}(\mathcal{E}) \cong \mathcal{B} \otimes_{\mathscr{O}_{X}} \operatorname{End}_{\mathscr{O}_{X}}(\mathcal{F}) .
$$

Definition 3.3. The Azumaya Brauer group of a scheme $X$ is the set of similarity classes of Azumaya algebras on $X$, with multiplication induced by tensor product of sheaves. We denote this group by $\mathrm{Br}_{\mathrm{Az}} X$.

The inverse of $[\mathcal{A}] \in \mathrm{Br}_{\mathrm{Az}} X$ is the class $\left[\mathcal{A}^{\mathrm{op}}\right]$ of the opposite algebra of $\mathcal{A}$; the identity element is $\left[\mathscr{O}_{X}\right]$ (see [Gro68, p. 47]).

Definition 3.4. The Brauer group of a scheme $X$ is $\operatorname{Br} X:=\mathrm{H}_{\text {êt }}^{2}\left(X, \mathbb{G}_{m}\right)$.

Remark 3.5. Note that if $k$ is a field, then $\mathrm{Br}_{\mathrm{Az}} \operatorname{Spec} k=\mathrm{Br} \operatorname{Spec} k=\mathrm{Br} k$, the usual Brauer group of a field.

For any scheme $X$ there is a natural inclusion

$$
\mathrm{Br}_{\mathrm{Az}} X \hookrightarrow \operatorname{Br} X ;
$$


see [Mil80, Theorem IV.2.5]. The following result of Gabber, a proof of which can be found in $[\mathrm{dJ}]$, determines the image of this injection for a scheme with some kind of polarization.

Theorem 3.6 (Gabber, de Jong). If $X$ is a scheme quasi-projective over a noetherian ring, then the natural map $\mathrm{Br}_{\mathrm{Az}} X \hookrightarrow \operatorname{Br} X$ induces an isomorphism

$$
\mathrm{Br}_{\mathrm{Az}} X \stackrel{\sim}{\rightarrow}(\mathrm{Br} X)_{\text {tors }}
$$

If $X$ is an integral scheme with function field $\mathbf{k}(X)$, then the inclusion $\operatorname{Spec} \mathbf{k}(X) \rightarrow X$ gives rise to a map $\operatorname{Br} X \rightarrow \operatorname{Br} \mathbf{k}(X)$ via functoriality of étale cohomology. If further $X$ is regular and quasi-compact then this induced map is injective; see [Mil80, Example III.2.22]. On the other hand, the group $\operatorname{Br} \mathbf{k}(X)$ is torsion, because it is a Galois cohomology group. These two facts imply the following corollary of Theorem 3.6.

Corollary 3.7. Let $X$ be a regular quasiprojective variety over a field. Then

$$
\operatorname{Br}_{A z} X \cong \operatorname{Br} X
$$

This corollary allows us to think of elements in "the" Brauer group of a nice $k$-variety either as Azumaya algebras or as étale cohomology classes. Each point of view has its advantages: the former is useful for computations, while the latter allows us to use theorems from étale cohomology to deduce things about the structure of the Brauer group.

3.3. The Brauer-Manin set II. Let $X$ be a nice variety over a global field $k$. For each $\mathcal{A} \in \operatorname{Br} X$ and each field extension $K / k$ there is a specialization map

$$
\mathrm{ev}_{\mathcal{A}}: X(K) \rightarrow \operatorname{Br} K, \quad x \mapsto \mathcal{A}_{x} \otimes_{\mathscr{O}_{X, x}} K
$$

These specialization maps may be put together to construct a pairing

$$
\phi: \operatorname{Br} X \times X\left(\mathbf{A}_{k}\right) \rightarrow \mathbb{Q} / \mathbb{Z}, \quad\left(\mathcal{A},\left(x_{v}\right)\right) \mapsto \sum_{v \in \Omega_{k}} \operatorname{inv}_{v}\left(\operatorname{ev}_{\mathcal{A}}\left(x_{v}\right)\right),
$$

where $\operatorname{inv}_{v}: \operatorname{Br} k_{v} \rightarrow \mathbb{Q} / \mathbb{Z}$ is the usual invariant map from local class field theory. The sum in (4) is in fact finite because for $\left(x_{v}\right) \in X\left(\mathbf{A}_{k}\right)$ we have $\operatorname{ev}_{\mathcal{A}}\left(x_{v}\right)=0 \in \mathrm{Br} k_{v}$ for all but finitely many $v$; see [Sko01, p. 101]. For $\mathcal{A} \in \operatorname{Br} X$ we obtain a commutative diagram

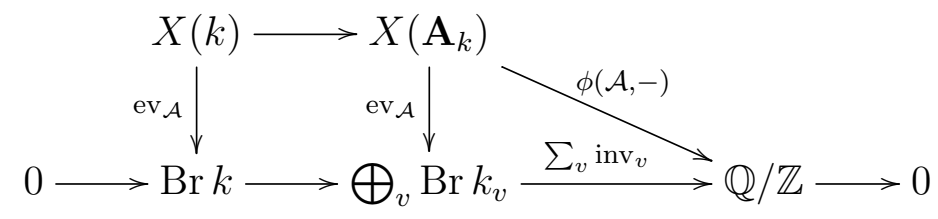

where the bottom row is the usual exact sequence from class field theory.

Manin's observation is that the kernel of the map $\phi(\mathcal{A},-)$ contains $X(k)$ (because the bottom row of the above commutative diagram is a complex), and thus an element $\mathcal{A} \in \operatorname{Br} X$ can be used to "carve out" a subset of $X\left(\mathbf{A}_{k}\right)$ that contains $X(k)$ :

$$
X\left(\mathbf{A}_{k}\right)^{\mathcal{A}}:=\left\{\left(x_{v}\right) \in X\left(\mathbf{A}_{k}\right): \phi\left(\mathcal{A},\left(x_{v}\right)\right)=0\right\} .
$$

Moreover, if $\mathbb{Q} / \mathbb{Z}$ is given the discrete topology, then the map $\phi(\mathcal{A},-): X\left(\mathbf{A}_{k}\right) \rightarrow \mathbb{Q} / \mathbb{Z}$ is continuous, so $X\left(\mathbf{A}_{k}\right)^{\mathcal{A}}$ is a closed subset of $X\left(\mathbf{A}_{k}\right)$; see [Har04, §3.1]. This shows that $\overline{X(k)} \subseteq X\left(\mathbf{A}_{k}\right)^{\mathcal{A}}$ 
Definition 3.8. Let $X$ be a nice variety over a global field $k$. We call

$$
X\left(\mathbf{A}_{k}\right)^{\mathrm{Br}}:=\bigcap_{\mathcal{A} \in \mathrm{Br} X} X(\mathbf{A})^{\mathcal{A}}
$$

the Brauer-Manin set of $X$.

The structure map $X \rightarrow$ Spec $k$ gives rise to a map $\operatorname{Br} k \rightarrow \operatorname{Br} X$, by functoriality. The group $\operatorname{Br}_{0} X:=\operatorname{im}(\operatorname{Br} k \rightarrow \operatorname{Br} X)$ is known as the subgroup of constant algebras. The exactness of the bottom row of (5) implies that if $\mathcal{A} \in \mathrm{Br}_{0} X$ then $X\left(\mathbf{A}_{k}\right)^{\mathcal{A}}=X\left(\mathbf{A}_{k}\right)$. This shows that to compute $\bigcap_{\mathcal{A} \in \operatorname{Br} X} X\left(\mathbf{A}_{k}\right)^{\mathcal{A}}$ it is enough to calculate the intersection over a set of representatives for the group $\operatorname{Br} X / \mathrm{Br}_{0} X$.

3.4. The Hochschild-Serre spectral sequence in étale cohomology. Let $X$ be a nice locally soluble variety over a global field $k$. If $\operatorname{Br} X_{k^{s}}=0$, then the Hochschild-Serre spectral sequence in étale cohomology provides a tool for computing the group $\operatorname{Br} X / \mathrm{Br} k$.

Let $K$ be a finite Galois extension of $k$, with Galois group $G$. The Hochschild-Serre spectral sequence

$$
E_{2}^{p, q}:=\mathrm{H}^{p}\left(G, \mathrm{H}_{\text {ét }}^{q}\left(X_{K}, \mathbb{G}_{m}\right)\right) \Longrightarrow \mathrm{H}_{\text {ét }}^{p+q}\left(X, \mathbb{G}_{m}\right)=: L^{p+q}
$$

gives rise to the usual "low-degree" long exact sequence

$$
0 \rightarrow E_{2}^{1,0} \rightarrow L^{1} \rightarrow E_{2}^{0,1} \rightarrow E_{2}^{2,0} \rightarrow \operatorname{ker}\left(L^{2} \rightarrow E_{2}^{0,2}\right) \rightarrow E_{2}^{1,1} \rightarrow E_{2}^{3,0}
$$

which in our case is

$$
\begin{aligned}
0 \rightarrow \operatorname{Pic} X & \rightarrow\left(\operatorname{Pic} X_{K}\right)^{G} \rightarrow \mathrm{H}^{2}\left(G, K^{*}\right) \rightarrow \operatorname{ker}\left(\operatorname{Br} X \rightarrow \operatorname{Br} X_{K}\right) \\
& \rightarrow \mathrm{H}^{1}\left(G, \operatorname{Pic} X_{K}\right) \rightarrow \mathrm{H}^{3}\left(G, K^{*}\right) .
\end{aligned}
$$

Taking the direct limit over all finite Galois extensions of $k$ gives the exact sequence

$$
\begin{aligned}
0 \rightarrow \operatorname{Pic} X & \rightarrow\left(\operatorname{Pic} X_{k^{s}}\right)^{\operatorname{Gal}\left(k^{s} / k\right)} \rightarrow \operatorname{Br} k \rightarrow \operatorname{ker}\left(\operatorname{Br} X \rightarrow \operatorname{Br} X_{k^{s}}\right) \\
& \rightarrow \mathrm{H}^{1}\left(\operatorname{Gal}\left(k^{s} / k\right), \operatorname{Pic} X_{k^{s}}\right) \rightarrow \mathrm{H}^{3}\left(\operatorname{Gal}\left(k^{s} / k\right), k^{s *}\right) .
\end{aligned}
$$

Furthermore, if $k$ is a global field, then $\mathrm{H}^{3}\left(\operatorname{Gal}\left(k^{s} / k\right), k^{s *}\right)=0$; this fact is due to Tatesee [NSW08, 8.3.11(iv), 8.3.17].

For each $v \in \Omega_{k}$, local solubility of $X$ gives a morphism Spec $k_{v} \rightarrow X$ that splits the base extension $\pi_{v}: X_{k_{v}} \rightarrow$ Spec $k_{v}$ of the structure map of $X$. Thus, by functoriality of the Brauer group, the natural maps $\pi_{v}^{*}: \operatorname{Br} k_{v} \rightarrow \operatorname{Br} X_{k_{v}}$ split for every $v \in \Omega_{k}$. The exactness of the bottom row of (5) then shows that the natural map $\operatorname{Br} k \rightarrow \operatorname{Br} X$ coming from the structure morphism of $X$ is injective. Moreover, if $X$ is a del Pezzo surface, then $\operatorname{Br} X_{k^{s}}=0$ and thus (7) gives rise to the short exact sequence

$$
0 \rightarrow \operatorname{Br} k \rightarrow \operatorname{Br} X \rightarrow \mathrm{H}^{1}\left(\operatorname{Gal}\left(k^{s} / k\right), \operatorname{Pic} X_{k^{s}}\right) \rightarrow 0
$$

and hence to an isomorphism

$$
\operatorname{Br} X / \operatorname{Br} k \stackrel{\sim}{\rightarrow} \mathrm{H}^{1}\left(\operatorname{Gal}\left(k^{s} / k\right), \operatorname{Pic} X_{k^{s}}\right) .
$$

If $K$ is a splitting field for $X$, i.e., a field extension $K$ of $k$ where a set of generators for Pic $X_{k^{s}}$ have representatives defined over $K$, then the inflation map

$$
\mathrm{H}^{1}\left(\operatorname{Gal}(K / k), \operatorname{Pic} X_{K}\right) \rightarrow \mathrm{H}^{1}\left(\operatorname{Gal}\left(k^{s} / k\right), \operatorname{Pic} X_{k^{s}}\right)
$$


is an isomorphism, because the cokernel maps into the first cohomology group of a free $\mathbb{Z}$-module with trivial action by a profinite group, which is trivial. Hence

$$
\operatorname{Br} X / \operatorname{Br} k \cong \mathrm{H}^{1}\left(\operatorname{Gal}(K / k), \operatorname{Pic} X_{K}\right) \text {. }
$$

Finally, we note that since $X\left(\mathbf{A}_{k}\right) \neq \emptyset$, if $H$ is a subgroup of $G$, then by (6) and the injectivity of the map $\operatorname{Br} k \rightarrow \operatorname{Br} X$, we know that

$$
\operatorname{Pic} X_{K^{H}} \stackrel{\sim}{\rightarrow}\left(\operatorname{Pic} X_{K}\right)^{H},
$$

where $K^{H}$ is the fixed field of $K$ by $H$.

In summary, one way of constructing Brauer-Manin obstructions on del Pezzo surfaces of small degree begins by computing the group $\mathrm{H}^{1}\left(\mathrm{Gal}(K / k)\right.$, $\left.\mathrm{Pic} X_{K}\right)$ on "reasonable" surfaces, and then tries to invert the isomorphism (9). Many authors have pursued this set of ideas, and not just for del Pezzo surfaces (see, for example, [Man74, CTCS80, CTSSD87, CTKS87, SD93, SD99, Bri02, KT04, Bri06, BBFL07, Cor07, KT08, Log08, VA08], to name but a few references).

3.5. A counterexample to weak approximation in degree 1 . We will use the remainder of this lecture to go through the details of a counterexample to weak approximation. We will deal with del Pezzo surfaces of degree 1 for two reasons: (1) we were explicitly asked to talk a little bit about our research in these lectures, and (2) the example shows that del Pezzo surfaces of degree 1 are not as scary as they may appear at first, if one is willing to use a computer to work out a little bit of algebra that is beyond what is reasonable to do with pencil and paper.

Let us quickly review what we know about del Pezzo surfaces of degree 1. First, recall their anticanonical model is a smooth sextic hypersurface in $\mathbb{P}_{k}(1,1,2,3):=\operatorname{Proj}(k[x, y, z, w])$, e.g.,

$$
w^{2}=z^{3}+A x^{6}+B y^{6}, \quad A, B \in k^{*} .
$$

Conversely, any smooth sextic $X$ in $\mathbb{P}_{k}(1,1,2,3)$ is a del Pezzo surface of degree 1 . The surface $X_{k^{s}}$ is isomorphic to the blow-up of $\mathbb{P}_{k^{s}}^{2}$ at 8 points in general position, so in particular, $\operatorname{Pic} X_{k^{s}} \cong \mathbb{Z}^{9}$.

Fix a primitive sixth root of unity $\zeta$ in $\overline{\mathbb{Q}}$. Our goal is to prove the following theorem.

Theorem 3.9 ([VA08]). Let $X$ be the del Pezzo surface of degree 1 over $k=\mathbb{Q}(\zeta)$ given by

$$
w^{2}=z^{3}+16 x^{6}+16 y^{6}
$$

in $\mathbb{P}_{k}(1,1,2,3)$. Then $X$ is $k$-minimal and there is a Brauer-Manin obstruction to weak approximation on $X$. Moreover, the obstruction arises from a cyclic algebra class in $\mathrm{Br} X / \mathrm{Br} k$.

In order to compute the Galois cohomology group $\mathrm{H}^{1}\left(\operatorname{Gal}\left(k^{s} / k\right)\right.$, $\left.\operatorname{Pic} X_{k^{s}}\right)$, we need an explicit description of the action of $\operatorname{Gal}\left(k^{s} / k\right)$ on $\operatorname{Pic} X_{k^{s}}$. Recall that Pic $X_{k^{s}}$ is generated by the exceptional curves of $X$. The following theorem, which can be deduced from work of Shioda on Mordell-Weil lattices (see [Shi90, Theorem 10.10] —we also give a different proof in [VA08, Theorem 1.2]), helps us compute the exceptional curves and a splitting field for a del Pezzo surface of degree 1.

Theorem 3.10. Let $X$ be a del Pezzo surface of degree 1 over a field $k$, given as a smooth sextic hypersurface $V(f(x, y, z, w))$ in $\mathbb{P}_{k}(1,1,2,3)$. Let

$$
\Gamma=V(z-Q(x, y), w-C(x, y)) \subseteq \mathbb{P}_{k^{s}}(1,1,2,3),
$$


where $Q(x, y)$ and $C(x, y)$ are homogenous forms of degrees 2 and 3 , respectively, in $k^{s}[x, y]$. If $\Gamma$ is a divisor on $X_{k^{s}}$, then it is an exceptional curve of $X$. Conversely, every exceptional curve on $X$ is a divisor of this form.

We explain how to use this theorem to compute the exceptional curves on the del Pezzo surface $X$ of Theorem 3.9. Let $a, b, c, r, s, t$ and $u$ be indeterminates, and let

$$
\begin{aligned}
& Q(x, y)=a x^{2}+b x y+c y^{2}, \\
& C(x, y)=r x^{3}+s x^{2} y+t x y^{2}+u y^{3} .
\end{aligned}
$$

The identity $C(x, y)^{2}=Q(x, y)^{3}+16 x^{6}+16 y^{6}$ gives

$$
\begin{aligned}
a^{3}-r^{2}+16 & =0, \\
3 a^{2} b-2 r s & =0, \\
3 a^{2} c+3 a b^{2}-2 r t-s^{2} & =0, \\
6 a b c+b^{3}-2 r u-2 s t & =0, \\
3 a c^{2}+3 b^{2} c-2 s u-t^{2} & =0, \\
3 b c^{2}-2 t u & =0, \\
c^{3}-u^{2}+16 & =0 .
\end{aligned}
$$

We can use Gröbner bases to solve this system of equations. We get 240 solutions, one for each exceptional curve of the surface. The action of $\operatorname{Gal}\left(k^{s} / k\right)$ can be read off from the coefficients of the equations of the exceptional curves. Let $s=\sqrt[3]{2}$, and consider the exceptional curves on $X$ given by

$$
\begin{aligned}
& E_{1}=V\left(z+2 s x^{2}, w-4 y^{3}\right), \\
& E_{2}=V\left(z-(-\zeta+1) 2 s x^{2}, w+4 y^{3}\right), \\
& E_{3}=V\left(z-2 \zeta s x^{2}+4 y^{2}, w-4 s(\zeta-2) x^{2} y-4(-2 \zeta+1) y^{3}\right), \\
& E_{4}=V\left(z+4 \zeta s x^{2}-2 s^{2}(2 \zeta-1) x y-4(-\zeta+1) y^{2},\right. \\
& \left.w-12 x^{3}-8 s(-\zeta-1) x^{2} y-12 \zeta s^{2} x y^{2}-4(-2 \zeta+1) y^{3}\right), \\
& E_{5}=V\left(z+4 \zeta s x^{2}-2 s^{2}(\zeta-2) x y-4 \zeta y^{2}\right. \\
& \left.w+12 x^{3}-8 s(2 \zeta-1) x^{2} y-12 s^{2} x y^{2}-4(-2 \zeta+1) y^{3}\right), \\
& E_{6}=V\left(z-2 s\left(-s^{2} \zeta+s^{2}-2 s+2 \zeta\right) x^{2}-2 s\left(2 s^{2} \zeta-2 s^{2}+3 s-4 \zeta\right) x y-2 s\left(-s^{2} \zeta+s^{2}-2 s+2 \zeta\right) y^{2}\right. \text {, } \\
& w-4\left(2 s^{2} \zeta-4 s^{2}+2 s \zeta+2 s-6 \zeta+3\right) x^{3}-4\left(-5 s^{2} \zeta+10 s^{2}-6 s \zeta-6 s+16 \zeta-8\right) x^{2} y \\
& \left.-4\left(5 s^{2} \zeta-10 s^{2}+6 s \zeta+6 s-16 \zeta+8\right) x y^{2}-4\left(-2 s^{2} \zeta+4 s^{2}-2 s \zeta-2 s+6 \zeta-3\right) y^{3}\right), \\
& E_{7}=V\left(z-2 s\left(-s^{2}-2 s \zeta+2 s+2 \zeta\right) x^{2}-2 s\left(-2 s^{2} \zeta+3 s+4 \zeta-4\right) x y-2 s\left(-s^{2} \zeta+s^{2}+2 s \zeta-2\right) y^{2}\right. \text {, } \\
& w-4\left(2 s^{2} \zeta+2 s^{2}+2 s \zeta-4 s-6 \zeta+3\right) x^{3}-4\left(10 s^{2} \zeta-5 s^{2}-6 s \zeta-6 s-8 \zeta+16\right) x^{2} y \\
& \left.-4\left(5 s^{2} \zeta-10 s^{2}-12 s \zeta+6 s+8 \zeta+8\right) x y^{2}-4\left(-2 s^{2} \zeta-2 s^{2}-2 s \zeta+4 s+6 \zeta-3\right) y^{3}\right), \\
& E_{8}=V\left(z-2 s\left(s^{2} \zeta+2 s \zeta+2 \zeta\right) x^{2}-2 s\left(2 s^{2}+3 s+4\right) x y-2 s\left(-s^{2} \zeta+s^{2}-2 s \zeta+2 s-2 \zeta+2\right) y^{2},\right. \\
& w-4\left(-4 s^{2} \zeta+2 s^{2}-4 s \zeta+2 s-6 \zeta+3\right) x^{3}-4\left(-5 s^{2} \zeta-5 s^{2}-6 s \zeta-6 s-8 \zeta-8\right) x^{2} y \\
& \left.-4\left(5 s^{2} \zeta-10 s^{2}+6 s \zeta-12 s+8 \zeta-16\right) x y^{2}-4\left(4 s^{2} \zeta-2 s^{2}+4 s \zeta-2 s+6 \zeta-3\right) y^{3}\right),
\end{aligned}
$$


as well as the exceptional curve

$$
E_{9}=V\left(z-2 \zeta_{3} s^{2} x y, w-4 x^{3}+4 y^{3}\right) .
$$

Then

$$
\operatorname{Pic} X_{k^{s}}=\operatorname{Pic} X_{K} \cong\left(\bigoplus_{i=1}^{8} \mathbb{Z}\left[E_{i}\right]\right) \oplus \mathbb{Z}[H]=\mathbb{Z}^{9}
$$

where $H=E_{1}+E_{2}+E_{9}$. The exceptional curves of $X$ are defined over $K:=k(\sqrt[3]{2})$. Let $\rho$ be a generator for the cyclic group $\operatorname{Gal}(K / k)$.

To invert the isomorphism (8), we will use the following diagram, whose individual maps we now explain:

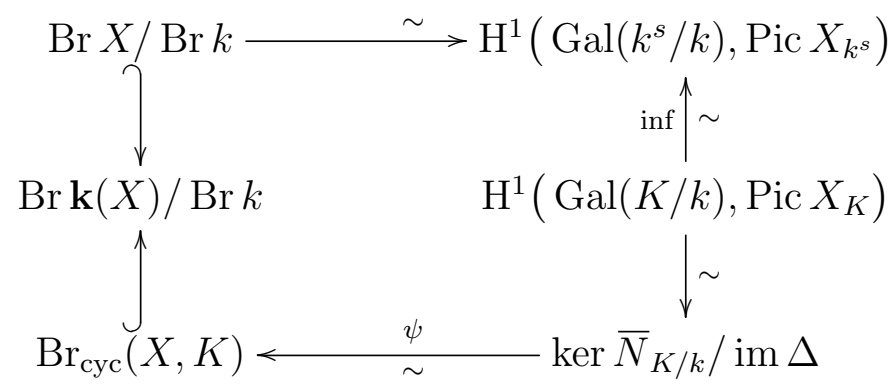

First, the map $\operatorname{Br} X \rightarrow \operatorname{Br} \mathbf{k}(X)$ is obtained by functoriality from the inclusion of the generic point $\operatorname{Spec} \mathbf{k}(X) \rightarrow X$ (see $\S 3.2$ ). Since $X$ is nice and $X\left(\mathbf{A}_{k}\right) \neq \emptyset$ (because $\left.X(k) \neq \emptyset\right)$, this map induces an injection $\operatorname{Br} X / \operatorname{Br} k \rightarrow \operatorname{Br} \mathbf{k}(X) / \operatorname{Br} k$.

Second, we have maps

$$
\begin{aligned}
\bar{N}_{K / k}: \operatorname{Pic} X_{K} & \rightarrow \operatorname{Pic} X & \Delta: \operatorname{Pic} X_{K} & \rightarrow \operatorname{Pic} X \\
{[D] } & \mapsto\left[D+{ }^{\rho} D+{ }^{\rho} D\right] & {[D] } & \mapsto\left[D-{ }^{\rho} D\right]
\end{aligned}
$$

and the isomorphism

$$
\mathrm{H}^{1}\left(\operatorname{Gal}(K / k), \operatorname{Pic} X_{K}\right) \cong \operatorname{ker} \bar{N}_{K / k} / \operatorname{im} \Delta
$$

comes from Tate cohomology, since $K$ is a cyclic extension of $k$.

To define the group $\operatorname{Br}_{\text {cyc }}(X, K)$, we first need to recall the notion of a cyclic algebra. If $L / k$ is a finite cyclic extension of fields of degree $n$, and if $\rho$ is a generator for $\operatorname{Gal}(L / k)$, then we let $L[x]_{\rho}$ be the "twisted" polynomial ring, where $\ell x=x^{\rho} \ell$ for all $\ell \in L$. Given $b \in k^{*}$, we write $(L / k, b)$ for the central simple $k$-algebra $L[x]_{\sigma} /\left(x^{n}-b\right)$. Let $f \in \mathbf{k}(X)^{*}$; since $X$ is geometrically integral, we have $\operatorname{Gal}\left(\mathbf{k}\left(X_{L}\right) / \mathbf{k}(X)\right) \cong \operatorname{Gal}(L / k)$, and hence we write $(L / k, f)$ instead of $\left(\mathbf{k}\left(X_{L}\right) / \mathbf{k}(X), f\right)$ for the cyclic algebra in $\operatorname{Br} \mathbf{k}(X)$ given by $f$.

We now define the group

$$
\operatorname{Br}_{\text {cyc }}(X, K):=\left\{\begin{array}{c}
\text { classes }[(K / k, f)] \text { in the image of the } \\
\operatorname{map} \operatorname{Br} X / \operatorname{Br} k \rightarrow \operatorname{Br} \mathbf{k}(X) / \operatorname{Br} k
\end{array}\right\} .
$$

The map $\psi$ in (10) is defined by

$$
\psi: \operatorname{ker} \bar{N}_{K / k} / \operatorname{im} \Delta \rightarrow \operatorname{Br}_{\text {cyc }}(X, K) \quad[D] \mapsto[(K / k, f)],
$$

where $f \in k(X)^{*}$ is any function such that $N_{K / k}(D)=(f)$. This map is a group isomorphism; see [VA08, Theorem 3.3]. 
With our explicit generators for Pic $K_{K}$ in hand, we use Magma [BCP97] to compute

$$
\operatorname{ker} \bar{N}_{K / k} / \operatorname{im} \Delta \cong(\mathbb{Z} / 3 \mathbb{Z})^{4} \text {. }
$$

The classes

$$
\begin{aligned}
\mathfrak{h}_{1} & =\left[E_{2}+2 E_{8}-H\right], & \mathfrak{h}_{2} & =\left[E_{5}+2 E_{8}-H\right], \\
\mathfrak{h}_{3} & =\left[E_{7}+2 E_{8}-H\right], & \mathfrak{h}_{4} & =\left[3 E_{8}-H\right]
\end{aligned}
$$

of Pic $X_{K}$ give a set of generators for this group.

Consider the divisor class $\mathfrak{h}_{1}-\mathfrak{h}_{2}=\left[E_{2}-E_{5}\right] \in$ Pic $X_{K}$. It gives rise to a cyclic algebra $\mathscr{A}:=(K / k, f) \in \operatorname{Br}_{\text {cyc }}(X, K)$, where $f \in k(X)^{*}$ is any function such that

$$
N_{K / k}\left(E_{2}-E_{5}\right)=(f),
$$

To wit, $f$ is a function with zeroes along

$$
E_{2}+{ }^{\rho} E_{2}+{ }^{\rho^{2}} E_{2}
$$

and poles along

$$
E_{5}+{ }^{\rho} E_{5}+{ }^{\rho^{2}} E_{5} .
$$

Using the explicit equations for $E_{2}$ and $E_{5}$ we find that

$$
f:=\frac{w+4 y^{3}}{w+(2 \zeta+2) z y+(-8 \zeta+4) y^{3}+12 x^{3}}
$$

does the job.

Recall that $X$ is given by $w^{2}=z^{3}+16 x^{6}+16 y^{6}$. Note that

$$
P_{1}=[1: 0: 0: 4] \text { and } P_{2}=[0: 1: 0: 4]
$$

are in $X(k)$. Let $\mathfrak{p}$ be the unique prime above 3 in $k$. We compute

$$
\operatorname{inv}_{\mathfrak{p}}\left(\mathscr{A}\left(P_{1}\right)\right)=0 \quad \text { and } \quad \operatorname{inv}_{\mathfrak{p}}\left(\mathscr{A}\left(P_{2}\right)\right)=1 / 3 .
$$

Let $P \in X\left(\mathbf{A}_{k}\right)$ be the point that is equal to $P_{1}$ at all places except $\mathfrak{p}$, and is $P_{2}$ at $\mathfrak{p}$. Then

$$
\sum_{v \in \Omega_{k}} \operatorname{inv}_{v}\left(\mathscr{A}\left(P_{v}\right)\right)=1 / 3
$$

so $P \in X\left(\mathbf{A}_{k}\right) \backslash X\left(\mathbf{A}_{k}\right)^{\mathrm{Br}}$ and $X$ is a counterexample to weak approximation.

\section{REFERENCES}

[BSD75] B. J. Birch and H. P. F. Swinnerton-Dyer, The Hasse problem for rational surfaces, J. Reine Angew. Math. 274/275 (1975), 164-174. $\uparrow 3$

[BCP97] W. Bosma, J. Cannon, and C. Playoust, The Magma algebra system. I. The user language, J. Symbolic Comput. 24 (1997), no. 3-4, 235-265. $\uparrow 3.5$

[Bri02] M. Bright, Computations on diagonal quartic surfaces, 2002. Ph. D. thesis, University of Cambridge. $\uparrow 3.4$

[Bri06] _ Brauer groups of diagonal quartic surfaces, J. Symbolic Comput. 41 (2006), no. 5, 544-558. $\uparrow 3.4$

[BBFL07] M. J. Bright, N. Bruin, E. V. Flynn, and A. Logan, The Brauer-Manin obstruction and Sh[2], LMS J. Comput. Math. 10 (2007), 354-377 (electronic). $\uparrow 3.4$

[Châ44] F. Châtelet, Variations sur un thème de H. Poincaré, Ann. Sci. École Norm. Sup. (3) 61 (1944), 249-300 (French). $\uparrow 2.1$

[CT72] J.-L. Colliot-Thélène, Surfaces de Del Pezzo de degré 6, C. R. Acad. Sci. Paris Sér. A-B 275 (1972), A109-A111 (French). $\uparrow 2.4$ 
[CT99] _ Points rationnels sur les variétés non de type général. Chapitre II: Surfaces Rationnelles (April 5, 1999). http://www.math.u-psud.fr/ colliot/Cours.ChapII.dvi. $\uparrow 2$

[CTCS80] J.-L. Colliot-Thélène, D. Coray, and J.-J. Sansuc, Descente et principe de Hasse pour certaines variétés rationnelles, J. Reine Angew. Math. 320 (1980), 150-191 (French). $\uparrow 1.2,1.5 .3,3.4$

[CTKS87] J.-L. Colliot-Thélène, D. Kanevsky, and J.-J. Sansuc, Arithmétique des surfaces cubiques diagonales, Diophantine approximation and transcendence theory, 1987, pp. 1-108. $\uparrow 3.4$

[CTS77] J.-L. Colliot-Thélène and J.-J. Sansuc, La descente sur une variété rationnelle définie sur un corps de nombres, C. R. Acad. Sci. Paris Sér. A-B 284 (1977), no. 19, A1215-A1218 (French, with English summary). $\uparrow 3$

[CTS80] _ La descente sur les variétés rationnelles, Journées de Géometrie Algébrique d'Angers, Juillet 1979, Sijthoff \& Noordhoff, Alphen aan den Rijn, 1980, pp. 223-237. 1 1.5.3

[CTS82] _ Sur le principe de Hasse et l'approximation faible, et sur une hypothèse de Schinzel, Acta Arith. 41 (1982), no. 1, 33-53. $\uparrow 1.5 .3$

[CTSSD87] J.-L. Colliot-Thélène, J.-J. Sansuc, and H. P. F. Swinnerton-Dyer, Intersections of two quadrics and Châtelet surfaces, J. Reine Angew. Math. 374 (1987), 72-168. $\uparrow 3.4$

[Coo88] K. R. Coombes, Every rational surface is separably split, Comment. Math. Helv. 63 (1988), no. $2,305-311 . \uparrow 1.7,1.8$

[Cor07] P. Corn, The Brauer-Manin obstruction on del Pezzo surfaces of degree 2, Proc. London Math. Soc. (3) 95 (2007), no. 3, 735-777. 33.4

[CO99] P. Cragnolini and P. A. Oliverio, Lines on del Pezzo surfaces with $K_{S}^{2}=1$ in characteristic $\neq 2$, Comm. Algebra 27 (1999), no. 3, 1197-1206. $\uparrow 1.5 .4,1.5 .4$

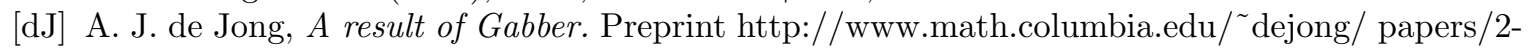
gabber.pdf. $\uparrow 3.2$

[Dem80] M. Demazure, Surfaces de Del Pezzo II, III, IV, V, Séminaire sur les Singularités des Surfaces, Lecture Notes in Mathematics, vol. 777, Springer, Berlin, 1980, pp. 23-69. $11.3,1.5$

[Enr97] F. Enriques, Sulle irrazionalità da cui può farsi dipendere la risoluzione d'un' equazione algebrica $f(x y z)=0$ con funzioni razionali di due parametri, Math. Ann. 49 (1897), no. 1, 1-23 (Italian). $\uparrow 2.5$

[GS06] P. Gille and T. Szamuely, Central simple algebras and Galois cohomology, Cambridge Studies in Advanced Mathematics, vol. 101, Cambridge University Press, Cambridge, 2006. $\uparrow 2.1,2.1$

[Gro68] A. Grothendieck, Le groupe de Brauer. I. Algèbres d'Azumaya et interprétations diverses, Dix Exposés sur la Cohomologie des Schémas, North-Holland, Amsterdam, 1968, pp. 46-66 (French). MR0244269 (39 \#5586a) $\uparrow 3.2$

[Har04] D. Harari, Weak approximation on algebraic varieties, Arithmetic of higher-dimensional algebraic varieties (Palo Alto, CA, 2002), 2004, pp. 43-60. $\uparrow 3.3$

[Har77] R. Hartshorne, Algebraic geometry, Springer-Verlag, New York, 1977. Graduate Texts in Mathematics, No. 52. $\uparrow 1.3,1.5 .1$

[Has09] B. Hassett, Rational surfaces over nonclosed fields, Arithmetic geometry, Clay Math. Proc., vol. 8, Amer. Math. Soc., Providence, RI, 2009, pp. 155-209. ^2.5, 2.6

[Isk79] V. A. Iskovskikh, Minimal models of rational surfaces over arbitrary fields, Izv. Akad. Nauk SSSR Ser. Mat. 43 (1979), no. 1, 19-43, 237 (Russian). $\uparrow 1.3,1.4$

[Kle05] Steven L. Kleiman, The Picard scheme, Fundamental algebraic geometry, Math. Surveys Monogr., vol. 123, Amer. Math. Soc., Providence, RI, 2005, pp. 235-321. $\uparrow 1.3$

[Kol96] J. Kollár, Rational curves on algebraic varieties, Ergebnisse der Mathematik und ihrer Grenzgebiete (3), vol. 32, Springer, Berlin, 1996. $\uparrow 1.5,1.5 .4,2.5$

[KT04] A. Kresch and Y. Tschinkel, On the arithmetic of del Pezzo surfaces of degree 2, Proc. London Math. Soc. (3) 89 (2004), no. 3, 545-569. $\uparrow 3,3.4$

[KT08] _ Two examples of BrauerManin obstruction to integral points, Bull. London Math. Soc. (May 21, 2008). To appear; doi:10.1112/blms/bdn081. $\uparrow 3,3.4$

[Lan54] S. Lang, Some applications of the local uniformization theorem, Amer. J. Math. 76 (1954), 362-374. $\uparrow 1.2$

[Lin40] C.-E. Lind, Untersuchungen über die rationalen Punkte der ebenen kubischen Kurven vom Geschlecht Eins, Thesis, University of Uppsala, 1940 (1940), 97 (German). $\uparrow 1.1$ 
[Log08] Adam Logan, The Brauer-Manin obstruction on del Pezzo surfaces of degree 2 branched along a plane section of a Kummer surface, Math. Proc. Cambridge Philos. Soc. 144 (2008), no. 3, 603-622. $\uparrow 3.4$

[Man71] Y. I. Manin, Le groupe de Brauer-Grothendieck en géométrie diophantienne, Actes du Congrès International des Mathématiciens (Nice, 1970), Gauthier-Villars, Paris, 1971, pp. 401-411. $\uparrow 3.1$

[Man74] Yu. I. Manin, Cubic forms: algebra, geometry, arithmetic, North-Holland Publishing Co., Amsterdam, 1974. $\uparrow 1.4,1.9,1.5,1.5 .2,1.5 .3,3.4$

[Mil80] J. S. Milne, Étale cohomology, Princeton Mathematical Series, vol. 33, Princeton University Press, Princeton, N.J., 1980. $\uparrow 3.2,3.2$

[NSW08] J. Neukirch, A. Schmidt, and K. Wingberg, Cohomology of number fields, 2nd ed., Grundlehren der Mathematischen Wissenschaften, vol. 323, Springer-Verlag, Berlin, 2008. $\uparrow 2.1,3.4$

[Nis55] H. Nishimura, Some remarks on rational points, Mem. Coll. Sci. Univ. Kyoto. Ser. A. Math. 29 (1955), 189-192. $\uparrow 1.2$

[Rei42] H. Reichardt, Einige im Kleinen überall lösbare, im Grossen unlösbare diophantische Gleichungen, J. Reine Angew. Math. 184 (1942), 12-18 (German). $\uparrow 1.1$

[RY00] Zinovy Reichstein and Boris Youssin, Essential dimensions of algebraic groups and a resolution theorem for G-varieties, Canad. J. Math. 52 (2000), no. 5, 1018-1056, DOI 10.4153/CJM-2000043-5. With an appendix by János Kollár and Endre Szabó. MR1782331 (2001k:14088) 11.2

[Ser88] J.-P. Serre, Algebraic groups and class fields, Graduate Texts in Mathematics, vol. 117, SpringerVerlag, New York, 1988. Translated from the French. MR918564 (88i:14041) $\uparrow 1.3$

[SB92] N. I. Shepherd-Barron, The rationality of quintic Del Pezzo surfaces - a short proof, Bull. London Math. Soc. 24 (1992), no. 3, 249-250. $\uparrow 2.5$

[Shi90] T. Shioda, On the Mordell-Weil lattices, Comment. Math. Univ. St. Paul. 39 (1990), no. 2, 211-240. $\uparrow 3.5$

[Sko93] Alexei N. Skorobogatov, On a theorem of Enriques-Swinnerton-Dyer, Ann. Fac. Sci. Toulouse Math. (6) 2 (1993), no. 3, 429-440 (English, with English and French summaries). MR1260765 (95b:14018) $\uparrow 2.5$

[Sko01] A. N. Skorobogatov, Torsors and rational points, Cambridge Tracts in Mathematics, vol. 144, Cambridge University Press, Cambridge, 2001. $\uparrow 1.2,3.3$

[SD62] H. P. F. Swinnerton-Dyer, Two special cubic surfaces, Mathematika 9 (1962), 54-56. $\uparrow 3$

[SD72] _ Rational points on del Pezzo surfaces of degree 5, Algebraic geometry, Oslo 1970 (Proc. Fifth Nordic Summer School in Math.), Wolters-Noordhoff, Groningen, 1972, pp. 287-290. $\uparrow 2.5$

[SD93] _ The Brauer group of cubic surfaces, Math. Proc. Cambridge Philos. Soc. 113 (1993), no. $3,449-460 . \uparrow 3.4$

[SD99] _ Brauer-Manin obstructions on some Del Pezzo surfaces, Math. Proc. Cambridge Philos. Soc. 125 (1999), no. 2, 193-198. $\uparrow 3.4$

[VA08] A. Várilly-Alvarado, Weak approximation on del Pezzo surfaces of degree 1, Adv. Math. 219 (2008), no. 6, 2123-2145. $\uparrow 3,3.4,3.9,3.5,3.5$

[VA09] Arithmetic of del Pezzo surfaces of degree 1, 2009. Ph. D. thesis, University of California at Berkeley. $\uparrow$ (document)

Department of Mathematics, Rice University, Houston, TX 77005, USA

E-mail address: varilly@rice.edu

$U R L:$ http://www.math.rice.edu/ av15 